\title{
OPEN Linear confinement of generalized KG-oscillator with a uniform magnetic field in Kaluza-Klein theory and Aharonov-Bohm effect
}

\author{
Faizuddin Ahmed
}

In this paper, we solve generalized KG-oscillator interacts with a uniform magnetic field in fivedimensional space-time background produced by topological defects under a linear confining potential using the Kaluza-Klein theory. We solve this equation and analyze an analogue of the AharonovBohm effect for bound states. We observe that the energy level for each radial mode depend on the global parameters characterizing the space-time, the confining potential, and the magnetic field which shows a quantum effect.

The first proposal of a unified theory of fundamental interactions was elaborated by Kaluza ${ }^{1,2}$ and Klein ${ }^{3,4}$ (see also, Ref. ${ }^{5}$ ). This new proposal established that the electromagnetism can be introduced through an extra (compactified) dimension in the space-time, where the spatial dimension becomes five-dimensional. This geometrical unification of gravitation and electromagnetism in five-dimensional version of general relativity gave some interesting results. The idea behind introducing additional space-time dimensions has found wide applications in quantum field theory ${ }^{6}$. Stationary cylindrically symmetric solutions to the five-dimensional Einstein and Einstein-Gauss-Bonnet equations has studied in Ref. ${ }^{7}$. Few examples of these solutions are the five-dimensional generalizations of cosmic string, chiral cosmic string ${ }^{8,9}$, and magnetic flux string ${ }^{10}$ space-times.

The Kaluza-Klein theory (KKT) has investigated in several branches of physics. For example, in Khaler fields ${ }^{11}$, in the presence of torsion ${ }^{12,13}$, in the Grassmannian context ${ }^{14-16}$, in the description of geometric phases in graphene $e^{17}$, in Kaluza-Klein reduction of a quadratic curvature model ${ }^{18}$, in the presence of fermions ${ }^{19-21}$, and in studies of the Lorentz symmetry violation ${ }^{22-24}$. In addition, the Kaluz-Klein theory has been studied in the relativistic quantum mechanics, for example, KG-oscillator on curved background in Ref. ${ }^{25}$, KG-oscillator field interacts with Cornell-type potential in Ref. ${ }^{26}$, generalized KG-oscillator in the background of magnetic cosmic string with scalar potential of Cornell-type in Ref. ${ }^{27}$, generalized KG-oscillator in the background of magnetic cosmic string with a linear confining potential in Ref. ${ }^{28}$, quantum dynamics of a scalar particle in the background of magnetic cosmic string and chiral cosmic string in Refs. ${ }^{29,30}$, bound states solution for a relativistic scalar particle subject to Coulomb-type potential in the Minkowski space-time in five dimensions in Ref. ${ }^{31}$, investigation of a scalar particle with position-dependent mass subject to a uniform magnetic field and quantum flux in the Minkowski space-time in five dimensions in Ref. ${ }^{32}$, and quantum dynamics of KG-scalar particle subject to linear and Coulomb-type central potentials in the five-dimensional Minkowski space-time ${ }^{33}$. Furthermore, effects of rotation on KG-scalar field subject Coulomb-type interaction and on KG-oscillator using Kaluza-Klein theory in the Minkowski space-time in five dimensions has also investigated ${ }^{34}$. In order to describe singular behavior for a system at large distances in a uniformly rotating frame on the clocks and on a rotating body, Landau et al. ${ }^{36}$ made a transformation such that it introduces a uniform rotation in the Minkowski space-time in cylindrical system. Non-inertial effects related to rotation have been investigated in several quantum systems, such as, in Dirac particle ${ }^{37}$, on a neutral particle ${ }^{38}$, on the Dirac oscillator ${ }^{39}$, in cosmic string space-time $e^{40,41}$, in cosmic string space-time with torsion ${ }^{42}$. Study of non-inertial effects on KG-oscillator within the Kaluza-Klein theory will be our next work.

The Klein-Gordon oscillator (KGO $)^{43,44}$ was inspired by the Dirac oscillator (DO) ${ }^{45}$ applied to spin- $\frac{1}{2}$ particle. Several authors have studied KGO on background space-times, for example, in cosmic string, Gödel-type spacetimes etc. (e.g. Refs. ${ }^{46-48}$ ). In the context of KKT, the KG-oscillator in five-dimensional cosmic string and magnetic cosmic string background ${ }^{25}$, under a Cornell-type potential in five-dimensional Minkowski space-time ${ }^{26}$ have investigated. In addition, generalized KGO on curved background space-time induced by a spinning cosmic string coupled to a magnetic field including quantum flux ${ }^{49}$, in magnetic cosmic string background under the 
effects of Cornell-type potential ${ }^{27}$ and a linear confining potential ${ }^{28}$ using KKT, in the presence of Coulomb-type potential in $(1+2)$-dimensions Gürses space-time ${ }^{50}$, in cosmic string space-time with a spacelike dislocation ${ }^{51}$, and in cosmic string space-time ${ }^{52}$ have investigated.

In this work, we study generalized KGO by introducing a uniform magnetic field in the cosmic string line element using $\mathrm{KKT}^{1-5}$ under the effects of a linear confining potential, and analyze a relativistic analogue of the Aharonov-Bohm effect for bound states. The Aharonov-Bohm effect ${ }^{53-56}$ is a quantum mechanical phenomena that describe phase shifts of the wave-function of a quantum particle due to the presence of a quantum flux produced by topological defects space-times. This effect has investigated by several authors in different branches of physics, such as, in Newtonian theory ${ }^{57}$, in bound states of massive fermions ${ }^{58}$, in scattering of dislocated wave-fronts $s^{59}$, on position-dependent mass system under torsion effects ${ }^{42,48,60}$, in bound states solution of spin- 0 scalar particles ${ }^{49}$. In addition, this effect has investigated using KKT with or without interactions of various kind in five-dimensional the Minkowski or cosmic string space-time background ${ }^{25,27-34}$.

\section{Interactions of generalized KGO with scalar potential using the KKT}

The basic idea of the Kaluz-Klein theory ${ }^{1-5,29}$ was to postulate one extra compactified space dimension and introducing pure gravity in new $(1+4)$-dimensional space-time. It turns out that the five-dimensional gravity manifests in our observable $(1+3)$-dimensional space-time as gravitational, electromagnetic and scalar filed. In this way, we can work with general relativity in five-dimensions. The information about the electromagnetism is given by introducing a gauge potential $A_{\mu}$ in the space-time ${ }^{26,29,30}$ as

$$
d s^{2}=-d t^{2}+d r^{2}+\alpha^{2} r^{2} d \phi^{2}+d z^{2}+\left[d y+\kappa A_{\mu}\left(x^{\mu}\right) d x^{\mu}\right]^{2},
$$

where $\mu=0,1,2,3, x^{0}=t$ is the time-coordinate, $x^{4}=y$ is the coordinate associated with fifth additional dimension having ranges $0<y<2 \pi a$ where, $a$ is the radius of the compact dimension of $y,\left(x^{1}=r, x^{2}=\phi, x^{3}=z\right)$ are the cylindrical coordinates with the usual ranges, and $\kappa$ is the gauge coupling or Kaluza constant ${ }^{29}$. The parameter $\alpha=(1-4 \mu)^{61}$ characterizing the wedge parameter where, $\mu$ is the linear mass density of the string. We assume the values of the parameter $\alpha$ lies in the range $0<\alpha<1$.

Based on Refs. ${ }^{25-27,29,31,32}$, we introduce a uniform magnetic field $B_{0}$ and quantum flux $\Phi$ through the lineelement of the cosmic string space-time (1) in the following form

$$
d s^{2}=-d t^{2}+d r^{2}+\alpha^{2} r^{2} d \phi^{2}+d z^{2}+\left[d y+\left(-\frac{1}{2} \alpha B_{0} r^{2}+\frac{\Phi}{2 \pi}\right) d \phi\right]^{2},
$$

where the gauge field given by

$$
A_{\phi}=\kappa^{-1}\left(-\frac{1}{2} \alpha B_{0} r^{2}+\frac{\Phi}{2 \pi}\right)
$$

gives rise to a uniform magnetic field $\vec{B}=\vec{\nabla} \times \vec{A}=-\kappa^{-1} B_{0} \hat{z}^{62}, \hat{z}$ is the unitary vector in the $z$-direction. Here $\Phi=$ const is quantum flux ${ }^{53,62}$ through the core of the topological defects ${ }^{63}$.

The relativistic quantum dynamics of spin- 0 scalar particle with a scalar potential $S(r)$ by modifying the mass term in the form $m \rightarrow m+S(r)$ as done in Refs. ${ }^{27,28,48}$ in five-dimensional case is described by ${ }^{26,31-33}$ :

$$
\left[\frac{1}{\sqrt{-g}} \partial_{M}\left(\sqrt{-g} g^{M N} \partial_{N}\right)-(m+S)^{2}\right] \Psi=0,
$$

where $M, N=0,1,2,3,4$, with $g=\operatorname{det} g=-\alpha^{2} r^{2}$ is the determinant of metric tensor $g_{M N}$ with $g^{M N}$ its inverse for the line element (2) and $m$ is rest mass of the particle.

To couple generalized Klein-Gordon oscillator with field, following change in the radial momentum operator is considered ${ }^{27,28,49-52,64}$

$$
\vec{p} \rightarrow \vec{p}+i m \Omega f(r) \hat{r} \quad, \quad \partial_{r} \rightarrow \partial_{r}+m \Omega f(r),
$$

where $\Omega$ is the oscillator frequency and we can write $\vec{p}^{2} \rightarrow(\vec{p}+i m \Omega f(r) \hat{r})(\vec{p}-i m \Omega f(r) \hat{r})$. Therefore, the KG-equation becomes

$$
\left[\frac{1}{\sqrt{-g}}\left(\partial_{M}+m \Omega X_{M}\right) \sqrt{-g} g^{M N}\left(\partial_{N}-m \Omega X_{N}\right)-(m+S)^{2}\right] \Psi=0,
$$

where $X_{M}=(0, f(r), 0,0,0)$.

For the metric (2)

$$
g^{M N}=\left(\begin{array}{ccccc}
-1 & 0 & 0 & 0 & 0 \\
0 & 1 & 0 & 0 & 0 \\
0 & 0 & \frac{1}{\alpha^{2} r^{2}} & 0 & -\frac{K A_{\phi}}{\alpha^{2} r^{2}} \\
0 & 0 & 0 & 1 & 0 \\
0 & 0 & -\frac{K A_{\phi}}{\alpha^{2} r^{2}} & 0 & 1+\frac{K^{2} A_{\phi}^{2}}{\alpha^{2} r^{2}}
\end{array}\right) .
$$

By considering the line-element (2) into the Eq. (6), we obtain the following differential equation: 


$$
\begin{gathered}
{\left[-\frac{\partial^{2}}{\partial t^{2}}+\frac{\partial^{2}}{\partial r^{2}}+\frac{1}{r} \frac{\partial}{\partial r}+\frac{1}{\alpha^{2} r^{2}}\left(\frac{\partial}{\partial \phi}-\kappa A_{\phi} \frac{\partial}{\partial y}\right)^{2}+\frac{\partial^{2}}{\partial z^{2}}+\frac{\partial^{2}}{\partial y^{2}}\right.} \\
\left.-m \Omega\left(f^{\prime}+\frac{f}{r}\right)-m^{2} \Omega^{2} f^{2}(r)-(m+S)^{2}\right] \Psi(t, r, \phi, z, y)=0 .
\end{gathered}
$$

Since the line-element (2) is independent of $t, \phi, z, x$. One can choose the following ansatz for the function $\Psi$ as:

$$
\Psi(t, r, \phi, z, y)=e^{i(-E t+l \phi+k z+q y)} \psi(r),
$$

where $E$ is the total energy of the particle, $l=0, \pm 1, \pm 2, \ldots \in \mathbf{Z}$, and $k, q$ are constants.

Substituting the ansatz (9) into the Eq. (8), we obtain the following equation:

$$
\begin{aligned}
& {\left[\frac{d^{2}}{d r^{2}}+\frac{1}{r} \frac{d}{d r}+E^{2}-k^{2}-q^{2}-m \Omega\left(f^{\prime}+\frac{f}{r}\right)-m^{2} \Omega^{2} f^{2}(r)-\frac{\left(l-K q A_{\phi}\right)^{2}}{\alpha^{2} r^{2}}\right] \psi(r)} \\
& \quad=(m+S)^{2} \psi(r) .
\end{aligned}
$$

Linear confining potential. In this work, we consider linear confining potential that studies in the confinement of quarks ${ }^{65}$, in the relativistic quantum mechanics ${ }^{28,47,48,66-71}$, and in atomic and molecular physics ${ }^{72}$. This potential is given by

$$
S(r)=\eta_{L} r,
$$

where $\eta_{L}$ is a constant that characterizes the linear confining potential.

Below, we choose two types of function $f(r)$ for the studies of generalized KG-oscillator in the considered relativistic system subject to linear confining potential.

Case A Cornell-type function $f(r)=b_{1} r+\frac{b_{2}}{r}$

Substituting Eqs. (3) and (11) into the Eq. (10) and using the above function, we obtain the following equation:

$$
\left[\frac{d^{2}}{d r^{2}}+\frac{1}{r} \frac{d}{d r}+\lambda-\frac{j^{2}}{r^{2}}-\omega^{2} r^{2}-b r\right] \psi(r)=0,
$$

where

$$
\begin{aligned}
\lambda & =E^{2}-k^{2}-q^{2}-m^{2}-2 m \omega_{c} \frac{\left(l-\frac{q \Phi}{2 \pi}\right)}{\alpha}-2 m \Omega b_{1}-2 m^{2} \Omega^{2} b_{1} b_{2}, \\
\omega & =\sqrt{m^{2} \omega_{c}^{2}+\eta_{L}^{2}+m^{2} \Omega^{2} b_{1}^{2}}, \\
j & =\sqrt{\frac{\left(l-\frac{q \Phi}{2 \pi}\right)^{2}}{\alpha^{2}}+m^{2} \Omega^{2} b_{2}^{2}}, \\
\omega_{c} & =\frac{q B_{0}}{2 m} \\
b & =2 m \eta_{L} .
\end{aligned}
$$

Introducing a new variable $\rho=\sqrt{\omega} r$, Eq. (12) becomes

$$
\left[\frac{d^{2}}{d \rho^{2}}+\frac{1}{\rho} \frac{d}{d \rho}+\zeta-\frac{j^{2}}{\rho^{2}}-\rho^{2}-\theta \rho\right] \psi(\rho)=0,
$$

where

$$
\zeta=\frac{\lambda}{\omega} \quad, \quad \theta=\frac{b}{\omega^{\frac{3}{2}}}
$$

Let us impose the requirement that the wave-function $\psi(\rho) \rightarrow 0$ for both $\rho \rightarrow 0$ and $\rho \rightarrow \infty$. Suppose the possible solution to Eq. (14) is

$$
\psi(\rho)=\rho^{j} e^{-\frac{1}{2}(\rho+\theta) \rho} H(\rho) .
$$

Substituting the solution Eq. (16) into the Eq. (14), we obtain

$$
H^{\prime \prime}(\rho)+\left[\frac{\gamma}{\rho}-\theta-2 \rho\right] H^{\prime}(\rho)+\left[-\frac{\beta}{\rho}+\Theta\right] H(\rho)=0,
$$

where 


$$
\begin{aligned}
& \gamma=1+2 j, \\
& \Theta=\zeta+\frac{\theta^{2}}{4}-2(1+j), \\
& \beta=\frac{\theta}{2}(1+2 j) .
\end{aligned}
$$

Equation (17) is the biconfluent Heun's differential equation $27,28,48,60,73,74$ and $H(\rho)$ is the Heun polynomials.

The above equation (17) can be solved by the Frobenius method. We consider the power series solution ${ }^{75}$

$$
H(\rho)=\sum_{i=0}^{\infty} c_{i} \rho^{i}
$$

Substituting the above power series solution into the Eq. (17), we obtain the following recurrence relation for the coefficients:

$$
c_{n+2}=\frac{1}{(n+2)(n+2+2 j)}\left[\{\beta+\theta(n+1)\} c_{n+1}-(\Theta-2 n) c_{n}\right] .
$$

And the various coefficients are

$$
\begin{aligned}
c_{1} & =\frac{\theta}{2} c_{0}, \\
c_{2} & =\frac{1}{4(1+j)}\left[(\beta+\theta) c_{1}-\Theta c_{0}\right] .
\end{aligned}
$$

We must truncate the power series by imposing the following two conditions $27,28,31-33,48,60$ :

$$
\begin{aligned}
\Theta & =2 n, \quad(n=1,2, \ldots) \\
c_{n+1} & =0 .
\end{aligned}
$$

By analyzing the condition $\Theta=2 n$, we get the following second degree expression of the energy eigenvalues $E_{n, l}$ :

$$
\begin{aligned}
& \frac{\lambda}{\omega}+\frac{\theta^{2}}{4}-2(1+j)=2 n \\
\Rightarrow & E_{n, l}= \pm\left\{k^{2}+q^{2}+m^{2}+2 \omega\left(n+1+\sqrt{\frac{\left(l-\frac{q \Phi}{2 \pi}\right)^{2}}{\alpha^{2}}+m^{2} \Omega^{2} b_{2}^{2}}\right)\right. \\
& \left.+2 m \omega_{c} \frac{\left(l-\frac{q \Phi}{2 \pi}\right)}{\alpha}-\frac{m^{2} \eta_{L}^{2}}{\omega^{2}}+2 m \Omega b_{1}\left(1+m \Omega b_{2}\right)\right\}^{\frac{1}{2}} .
\end{aligned}
$$

Note that the Eq. (23) does not represent the general expression for eigenvalues. One can obtain the individual energy eigenvalues one by one, that is, $E_{1}, E_{2}, E_{3}, \ldots$ by imposing the additional recurrence condition $C_{n+1}=0$ on the eigenvalue as done in Refs. ${ }^{27,28,48,60}$. For $n=1$, we have $\Theta=2$ and $c_{2}=0$ which implies from Eq. (21)

$$
\begin{aligned}
& c_{1}=\frac{2}{\beta+\theta} c_{0} \Rightarrow \frac{\theta}{2}=\frac{2}{\beta+\theta} \\
& \omega_{1, l}=\left[\frac{b^{2}}{8}(3+2 j)\right]^{\frac{1}{3}}=\left[\frac{m^{2} \eta_{1 L}^{2}}{2}(3+2 j)\right]^{\frac{1}{3}}
\end{aligned}
$$

a constraint on the parameter $\omega_{1, l}$. The relation given in Eq. (24) gives the value of the parameter $\omega_{1, l}$ that permit us to construct a first degree polynomial solution of $H(\rho)$ for the radial mode $n=1$. Note that the parameter $\omega_{1, l}$ depends on the linear confining potential $\eta_{L}$ and its value changes for each quantum number $\{n, l\}$, so we have labeled $\omega \rightarrow \omega_{n, l}$ and $\eta_{L} \rightarrow \eta_{1 L}$. Besides, we have adjusted the magnetic field $B_{0}^{1, l}$ and the linear confining potential $\eta_{1 L}$ such that Eq. (24) can be satisfied and we have simplified by labelling:

$$
\omega_{c}^{1, l}=\frac{1}{m} \sqrt{\omega_{1, l}^{2}-\eta_{1 L}^{2}-m^{2} \Omega^{2} b_{1}^{2}} \leftrightarrow B_{0}^{1, l}=\frac{2}{q} \sqrt{\omega_{1, l}^{2}-\eta_{1 L}^{2}-m^{2} \Omega^{2} b_{1}^{2}} .
$$

It is noteworthy that the allowed value of the magnetic field $B_{0}^{1, l}$ for lowest state of the system given by (25) is defined for the radial mode $n=1$. We can note from Eq. (25) that the magnetic field $B_{0}$ depends on the quantum numbers $\{n, l\}$ of the relativistic system which shows a quantum effect.

Therefore, the ground state energy level for $n=1$ is given by 


$$
\begin{aligned}
E_{1, l}= & \pm\left\{k^{2}+q^{2}+m^{2}+2 \omega_{1, l}\left(2+\sqrt{\frac{\left(l-\frac{q \Phi}{2 \pi}\right)^{2}}{\alpha^{2}}+m^{2} \Omega^{2} b_{2}^{2}}\right)\right. \\
& \left.+2 m \omega_{c}^{1, l} \frac{\left(l-\frac{q \Phi}{2 \pi}\right)}{\alpha}-\frac{m^{2} \eta_{L}^{2}}{\omega_{1, l}^{2}}+2 m \Omega b_{1}\left(1+m \Omega b_{2}\right)\right\}^{\frac{1}{2}}
\end{aligned}
$$

And the radial wave-functions is

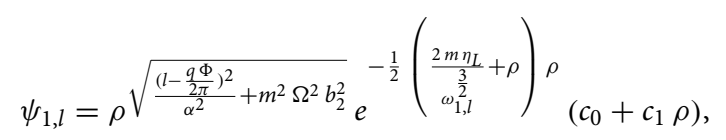

where

$$
c_{1}=\frac{1}{\sqrt{\frac{3}{2}+\sqrt{\frac{\left(l-\frac{q \Phi}{2 \pi}\right)^{2}}{\alpha^{2}}+m^{2} \Omega^{2} b_{2}^{2}}}} c_{0} .
$$

Then, by substituting the magnetic field (25) into the Eq. (26), one can obtain the allowed values of the relativistic energy level for the radial mode $n=1$ of the system. As the values of the wedge parameter $\alpha$ are in the ranges $0<\alpha<1$, thus, the degeneracy of the energy is broken and shifted the energy level in comparison to the case of five-dimensional Minkowski space-time.

Case B Coulomb-type function $f(r)=\frac{b_{2}}{r}$

In that case, the radial wave-equation (12) becomes

$$
\left[\frac{d^{2}}{d r^{2}}+\frac{1}{r} \frac{d}{d r}+\tilde{\lambda}-\frac{j^{2}}{r^{2}}-\tilde{\omega}^{2} r^{2}-b r\right] \psi(r)=0
$$

where

$$
\begin{aligned}
& \tilde{\lambda}=E^{2}-k^{2}-q^{2}-m^{2}-2 m \omega_{c} \frac{\left(l-\frac{q \Phi}{2 \pi}\right)}{\alpha}, \\
& \tilde{\omega}=\sqrt{m^{2} \omega_{c}^{2}+\eta_{L}^{2}} .
\end{aligned}
$$

Introducing a new variable $\rho=\sqrt{\tilde{\omega}} r$, Eq. (12) becomes

$$
\left[\frac{d^{2}}{d \rho^{2}}+\frac{1}{\rho} \frac{d}{d \rho}+\tilde{\zeta}-\frac{j^{2}}{\rho^{2}}-\rho^{2}-\tilde{\theta} \rho\right] \psi(\rho)=0
$$

where

$$
\tilde{\zeta}=\frac{\tilde{\lambda}}{\tilde{\omega}} \quad, \quad \tilde{\theta}=\frac{b}{\tilde{\omega}^{\frac{3}{2}}}
$$

Let the possible solution to Eq. (31) is

$$
\psi(\rho)=\rho^{j} e^{-\frac{1}{2}(\rho+\tilde{\theta}) \rho} H(\rho) .
$$

Substituting solution Eq. (33) into the Eq. (31), we obtain

$$
H^{\prime \prime}(\rho)+\left[\frac{\gamma}{\rho}-\tilde{\theta}-2 \rho\right] H^{\prime}(\rho)+\left[-\frac{\tilde{\beta}}{\rho}+\tilde{\Theta}\right] H(\rho)=0,
$$

where

$$
\begin{aligned}
& \tilde{\Theta}=\tilde{\zeta}+\frac{\tilde{\theta}^{2}}{4}-2(1+j), \\
& \tilde{\beta}=\frac{\tilde{\theta}}{2}(1+2 j) .
\end{aligned}
$$

Equation (34) is the biconfluent Heun's differential equation ${ }^{27,28,48,60,73,74}$ and $H(\rho)$ is the Heun polynomials.

Substituting the above power series solution (19) into the Eq. (34), we obtain the following recurrence relation for the coefficients:

$$
c_{n+2}=\frac{1}{(n+2)(n+2+2 j)}\left[\{\tilde{\beta}+\tilde{\theta}(n+1)\} c_{n+1}-(\tilde{\Theta}-2 n) c_{n}\right] .
$$


And the various coefficients are

$$
\begin{aligned}
c_{1} & =\frac{\tilde{\theta}}{2} c_{0}, \\
c_{2} & =\frac{1}{4(1+j)}\left[(\tilde{\beta}+\tilde{\theta}) c_{1}-\tilde{\Theta} c_{0}\right] .
\end{aligned}
$$

We must truncate the power series by imposing the following two conditions $s^{27,28,31-33,48,60}$ :

$$
\begin{aligned}
\tilde{\Theta} & =2 n, \quad(n=1,2, \ldots) \\
c_{n+1} & =0 .
\end{aligned}
$$

By analyzing the condition $\tilde{\Theta}=2 n$, we get the following second degree expression of the energy eigenvalues $E_{n, l}$ :

$$
\begin{aligned}
& \frac{\tilde{\lambda}}{\tilde{\omega}}+\frac{\tilde{\theta}^{2}}{4}-2(1+j)=2 n \\
\Rightarrow & E_{n, l}= \pm\left\{k^{2}+q^{2}+m^{2}+2 \tilde{\omega}\left(n+1+\sqrt{\frac{\left(l-\frac{q \Phi}{2 \pi}\right)^{2}}{\alpha^{2}}+m^{2} \Omega^{2} b_{2}^{2}}\right)\right. \\
& \left.+2 m \omega_{c} \frac{\left(l-\frac{q \Phi}{2 \pi}\right)}{\alpha}-\frac{m^{2} \eta_{L}^{2}}{\tilde{\omega}^{2}}\right\}^{\frac{1}{2}} .
\end{aligned}
$$

Following the similar technique as done earlier, we want to find the individual energy level and wave-function. For example $n=1$ we have $\Theta=2$ and $c_{2}=0$ which implies from Eq. (21)

$$
\begin{aligned}
c_{1} & =\frac{2}{\tilde{\beta}+\tilde{\theta}} c_{0} \Rightarrow \frac{\tilde{\theta}}{2}=\frac{2}{\tilde{\beta}+\tilde{\theta}} \\
\tilde{\omega}_{1, l} & =\left[\frac{b^{2}}{8}(3+2 j)\right]^{\frac{1}{3}}
\end{aligned}
$$

a constraint on the parameter $\tilde{\omega}_{1, l}$. The magnetic field $B_{0}^{1, l}$ is so adjusted that Eq. (24) can be satisfied and we have simplified by labelling:

$$
\omega_{c}^{1, l}=\frac{1}{m} \sqrt{\tilde{\omega}_{1, l}^{2}-\eta_{L}^{2}} \leftrightarrow B_{0}^{1, l}=\frac{2}{q} \sqrt{\tilde{\omega}_{1, l}^{2}-\eta_{L}^{2}} .
$$

We can see from Eq. (41) that the possible values of the magnetic field $B_{0}$ depend on the quantum numbers $\{n, l\}$ of the system as well as on the confining potential parameter.

Therefore, the ground state energy level for $n=1$ is given by

$$
\begin{aligned}
E_{1, l}= & \pm\left\{k^{2}+q^{2}+m^{2}+2 \tilde{\omega}_{1, l}\left(2+\sqrt{\frac{\left(l-\frac{q \Phi}{2 \pi}\right)^{2}}{\alpha^{2}}+m^{2} \Omega^{2} b_{2}^{2}}\right)\right. \\
& \left.+2 m \omega_{c}^{1, l} \frac{\left(l-\frac{q \Phi}{2 \pi}\right)}{\alpha}-\frac{m^{2} \eta_{L}^{2}}{\tilde{\omega}_{1, l}^{2}}\right\}^{\frac{1}{2}} .
\end{aligned}
$$

And the radial wave-functions is

$$
\psi_{1, l}=\rho \sqrt{\frac{\left(l-\frac{q \Phi}{2 \pi}\right)^{2}}{\alpha^{2}}+m^{2} \Omega^{2} b_{2}^{2}} e^{-\frac{1}{2}\left(\frac{2 m \eta_{L}}{\tilde{\omega}_{1, l}^{2}}+\rho\right) \rho}\left(c_{0}+c_{1} \rho\right),
$$

where

$$
c_{1}=\frac{1}{\sqrt{\frac{3}{2}+\sqrt{\frac{\left(l-\frac{q \Phi}{2 \pi}\right)^{2}}{\alpha^{2}}+m^{2} \Omega^{2} b_{2}^{2}}}} c_{0} .
$$

Then, by substituting the real solution from Eq. (40) into the Eq. (41), it is possible to obtain the allowed values of the relativistic energy levels for the radial mode $n=1$ of the system. We can see that the lowest energy state defined by Eqs. (40)-(41) plus the expression given in Eqs. (42)-(44) is for the radial mode $n=1$, instead of $n=0$. This effect arises due to the presence of linear confining potential in the relativistic system. Since the wedge parameter $\alpha$ are in the ranges $0<\alpha<1$, thus, the degeneracy of the relativistic energy eigenvalue here also is broken and shifted the energy level in comparison to the case of five-dimensional Minkowski space-time. 


\section{Conclusions}

In this work, we have investigated generalized Klein-Gordon oscillator with a uniform magnetic field subject to a linear confining potential in a topological defect five-dimensional space-time in the context of Kaluza-Klein theory. Linear confining potential has many applications such as confinement of quarks in particle physics and other branches of physics including relativistic quantum mechanics. For suitable total wave-function, we have derived the radial wave-equation for a Cornell-type function in "Linear confining potential" and finally reached a biconfluent Heun's differential equation form. By power series method we have solved this equation and by imposing condition we have obtained the non-compact expression of the energy eigenvalues (23). By imposing the recurrence condition $c_{n+1}=0$ for each radial mode, for example $n=11$, we have obtained the lowest state energy level and wave-function by Eqs. (26)-(28), and others are in the same way.

In "Linear confining potential", we have considered a Coulomb-type function on the same relativistic system with a linear confining potential. Here also we have reached a biconfluent Heun's equation form and following the similar technique as done earlier, we have obtained the non-compact expression of the energy eigenvalues (39). By imposing the additional recurrence condition $c_{n+1}=0$ on the eigenvalue, one can obtained the individual energy level and the corresponding wave-function, as for example, for the radial mode $n=1$ by Eqs. (42)-(44), and others are in the same way. In "Linear confining potential", we have seen that the presence of linear confining potential allow the formation of bound states solution of the considered relativistic system and hence, the lowest energy state is defined by the radial mode $n=1$, instead of $n=0$. Also in gravitation and cosmology, the values of the wedge parameter $\alpha$ are in the ranges $0<\alpha<1$, and thus, the degeneracy of each energy level is broken and shifted the relativistic energy level in comparison to the case of five-dimensional Minkowski space-time. When we tries to analyze $c_{n+1}=0$ for the radial mode $n=1$, an observation is noted, where certain parameter is constraint, for example, $\omega_{1, l}$ that appears in Eq. (24) in "Linear confining potential" (Case A) depend on the quantum number $\{n, l\}$ of the system as well as on linear confining potential $\eta_{L}$. Another interesting observation that we made in this work is the quantum effect which arises due to the dependence of the magnetic field $B_{0}^{n, l}$ on the quantum number $\{n, l\}$ of the relativistic system.

We have observed in this work that the angular momentum number $l$ of the system is shifted, $l \rightarrow l_{0}=\frac{1}{\alpha}\left(l-\frac{q \Phi}{2 \pi}\right)$, an effective angular quantum number. Therefore, the relativistic energy eigenvalues depends on the geometric quantum phase ${ }^{53,62}$. Thus, we have that, $E_{n, l}\left(\Phi+\Phi_{0}\right)=E_{n, l \mp \tau}(\Phi)$, where $\Phi_{0}= \pm \frac{2 \pi}{q} \tau$ with $\tau=0,1,2, \ldots$. This dependence of the relativistic energy level on the geometric quantum phase gives us a relativistic analogue of the Aharonov-Bohm effect for bound states.

The Kaluza-Klein theory lead to many new unified field theories, for example, connecting this theory with supergravity resulted in improved supersymmetric Kaluza-Klein theory, multi-dimensional unified theories using the idea of Kaluza-Klein theory to postulate extra (compactified) space dimensions, superstring theory as well as the M-theory ${ }^{75}$ model using the Kaluza-Klein theory in higher dimensions. In addition, this theory has growing interest because of some interesting observations made in Ref. ${ }^{76}$. Authors pointed out that the fivedimensional theory yields a geometrical interpretation of the electromagnetic field and electric charge. The relation of the five-dimensional KKT with the structure of fiber bundles was first made in Ref. ${ }^{77}$ and the relationship between principal fiber bundles and higher dimensional theories is in $^{78}$.

Received: 13 October 2020; Accepted: 15 December 2020

Published online: 18 January 2021

\section{References}

1. Kaluza, T. On the problem of unity in physics. Sitzungsber. Preuss. Akad. Wiss. Berlin (Math. Phys.) K 1, 966 (1921).

2. Kaluza, Th. On the problem of unity in physics. Int. J. Mod. Phys. D 27, 1870001 (2018).

3. Klein, O. Quantentheorie und fünfdimensionale relativitätstheorie. Zeitschrift fur Physik 37, 895 (1926).

4. Klein, O. Quantentheorie und fünfdimensionale relativitätstheorie. Nature 118, 516 (1927).

5. Muta, T. An Introduction to Kaluza-Klein Theories. (ed. Lee, H. C.) (World Scientific publishing Co Pvt. Litd, Singapore, 1984).

6. Green, M. B., Schwarz, J. H., \& Witten, E. Superstring Theory, vol. 1-2 (Cambridge University Press, Cambridge, 1987).

7. Ainouy, M. A. \& Clement, G. Kaluz-Klein and Gauss-Bonnet cosmic strings. Class. Quantum Grav. 13, 2635 (1996).

8. Galtsov, D. V. \& Letelier, P. S. Spinning strings and cosmic dislocations. Phys. Rev. D 47, 9273 (1993).

9. Letelier, P. S. Spinning strings as torsion line spacetime defects. Class. Quantum Grav. 12, 471 (1995).

10. Guimaraes, M. E. X. Semiclassical effects induced by Aharonov-Bohm interaction between a cosmic string and a scalar field. Phys. Lett. B 398, 281 (1997).

11. Benn, I. M. \& Tucker, R. W. Kahler fields and five-dimensional Kaluz-Klein theory. J. Phys. A : Math. Gen. 16, L123 (1983).

12. German, G. On Kaluz-Klein theory with torsion. Class. Quantum Grav. 2, 455 (1985).

13. Wu, Y.-S. \& Zee, A. Massless fermions and Kaluz-Klein theory with torsion. J. Math. Phys. 25, 2696 (1984).

14. Ellicott, P. \& Toms, D. J. Grassmannian Kaluz-Klein theory. Class. Quantum Grav. 6, 1033 (1989).

15. Delbourgo, R., Twisk, S. \& Zhang, R. B. Grand unification and Grassmannian Kaluz-Klein theory. Mod. Phys. Lett. A 3, 1073 (1988).

16. Delbourgo, R. \& Zhang, R. B. Grassmannian Kaluz-Klein theory and the standard model. Phys. Rev. D 38, 2490 (1988).

17. Bakke, K., Yu, A. \& Petrov, C. F. A Kaluz-Klein description of geometric phases in graphene. Ann. Phys. 327, 2946 (2012).

18. Baskal, S. \& Kuyrukcu, H. Kaluz-Klein reduction of a quadratic curvature model. Gen. Relativ. Gravit. 45, 359 (2013).

19. Bailin, D. \& Love, A. Kaluz-Klein theories. Rep. Prog. Phys. 50, 1087 (1987).

20. Macias, A. \& Dehnen, H. Dirac field in the five-dimensional Kaluz-Klein theory. Class. Quantum Grav. 8, 203 (1991).

21. Ichinose, S. Fermions in Kaluz-Klein and Randall-Sundrum theories. Phys. Rev. D 66, 104015 (2002).

22. Carroll, S. M. \& Tam, H. Aether compactification. Phys. Rev. D 78, 044047 (2008).

23. Gomes, M., Nascimento, J. R., Petrov, A. Y. \& da Silva, A. J. Aetherlike Lorentz-breaking actions. Phys. Rev. D 81, 045018 (2010).

24. BaetaScarpelli, A. P., Mariz, T., Nascimento, J. R. \& Petrov, A. Y. Four-dimensional aether-like Lorentz-breaking QED revisited and problem of ambiguities. Eur. Phys. J. C 73, 2526 (2013).

25. de Carvalho, J. A. M. M., Carvalho, C. E. \& Furtado, C. Klein-Gordon oscillator in Kaluz-Klein theory. Eur. Phys. J. C 76, 365 (2016). 
26. Leite, E. V. B., Belich, H. \& Vitória, R. L. L. Klein-Gordon Oscillator Under the Effects of the Cornell-Type Interaction in the Kaluz-Klein Theory. Braz. J. Phys. 50, 744 (2020).

27. Ahmed, F. Effects of Kaluz-Klein Theory and potential on a generalized Klein-Gordon oscillator in the cosmic string space-time. Adv. High Energy Phys. 2020, 8107025 (2020).

28. Ahmed, F. The generalized Klein-Gordon oscillator in the background of cosmic string space-time with a linear potential in the Kaluz-Klein theory. Eur. Phys. J. C 80, 211 (2020).

29. Furtado, C., Moraes, F. \& Bezerra, V. B. Global effects due to cosmic defects in Kaluz-Klein theory. Phys. Rev. D 59, 107504 (1999).

30. Furtado, C., Bezerra, V. B. \& Moraes, F. Aharonov-Bohm effect for bound states in Kaluz-Klein theory. Mod. Phys. Lett A 15, 253 (2000).

31. Leite, E. V. B., Belich, H. \& Bakke, K. Aharonov-Bohm effect for bound states on the confinement of a relativistic scalar particle to a coulomb-type potential in Kaluz-Klein theory. Adv. High Energy Phys. 2015, 925846 (2015).

32. Leite, E. V. B., Belich, H. \& Vitória, R. L. L. Effects of the Cornell-type potential on a position-dependent mass system in KaluzKlein theory. Adv. High Energy Phys. 2019, 6740360 (2019).

33. Leite, E. V. B., Vitória, R. L. L. \& Belich, H. Effects of the Kaluza Klein theory on a Klein Gordon particle with position-dependent mass. Mod. Phys. A 34, 1950319 (2019).

34. Leite, E. V. B., Belich, H. \& Vitória, R. L. L. Effects of rotation on a scalar field in a Kaluz-Klein theory. Mod. Phys. Lett. A 35, $2050283(2020)$.

35. Ahmed, F. Spin-0 scalar particle interacts with scalar potential in the presence of magnetic field and quantum flux under the effects of KKT in 5D cosmic string space-time. Mod. Phys. Lett. A. https://doi.org/10.1142/S0217732321500048.

36. Landau, L. D. \& Lifshitz, E. M. The Classical Theory of Fields, Course of Theoretical Physics Vol. 2 (Elsevier, New York, 1980).

37. Hehl, F. W. \& Ni, W.-T. Inertial effects of a Dirac particle. Phys. Rev. D 42, 2045 (1990).

38. Bakke, K. Relativistic bounds states for a neutral particle confined to a parabolic potential induced by noninertial effects. Phys. Lett. A 374, 4642 (2010).

39. Strange, P. \& Ryder, L. H. The Dirac oscillator in a rotating frame of reference. Phys. Lett. A 380, 3465 (2016).

40. Castro, L. B. Noninertial effects on the quantum dynamics of scalar bosons. Eur. Phys. J. C 76, 61 (2016).

41. Santos, L. C. N. \& Barros, C. C. Jr. Relativistic quantum motion of spin-0 particles under the influence of noninertial effects in the cosmic string spacetime. Eur. Phys. J. C 78, 13 (2018).

42. Ahmed, F. Aharonov-Bohm and non-inertial effects on a Klein-Gordon oscillator with potential in the cosmic string space-time with a spacelike dislocation. Chin. J. Phys. 66, 587 (2020).

43. Bruce, S. \& Minning, P. The Klein-Gordon oscillator. II Nuovo Cimento A 106, 711 (1993).

44. Dvoeglazov, V. V. Comment on the Klein-Gordon oscillator by S. Bruce and P. Minning. II Nuovo Cimento A 107, 1413 (1994).

45. Moshinsky, M. The Dirac oscillator. J. Phys. A : Math. Gen. 22, L817 (1989).

46. Boumali, A. \& Messai, N. Klein-Gordon oscillator under a uniform magnetic field in cosmic string space-time. Can. J. Phys. 92, 1460 (2014).

47. Wang, Z., Long, Z., Long, C. \& Wu, M. Relativistic quantum dynamics of a spinless particle in the Som-Raychaudhuri spacetime. Eur. Phys. J. Plus 130, 36 (2015).

48. Ahmed, F. Klein-Gordon Oscillator in the Presence of External Fields in a Cosmic Space-Time with a Space-Like Dislocation and Aharonov-Bohm Effect. Adv. High Energy Phys. 2020, 5691025 (2020).

49. Ahmed, F. Aharonov-Bohm effect on a generalized Klein-Gordon oscillator with uniform magnetic field in a spinning cosmic string space-time. EPL 130, 40003 (2020).

50. Ahmed, F. The generalized Klein-Gordon oscillator with Coulomb-type potential in (1+2)-dimensions Gürses space-time. Gen. Relativ. Gravit. 51, 69 (2019).

51. Lütfüoğlu, B. C., Kříž, J., Sedaghatnia, P. \& Hassanabadi, H. The generalized Klein-Gordon oscillator in a cosmic space-time with a space-like dislocation and the Aharonov-Bohm effect. Eur. Phys. J. Plus 135, 691 (2020).

52. Deng, L.-F., Long, C.-Y., Long, Z.-W. \& Xu, T. The generalized K-G oscillator in the cosmic string space-time. Eur. Phys. J. Plus 134, 355 (2019).

53. Aharonov, Y. \& Bohm, D. Significance of Electromagnetic Potentials in the Quantum Theory. Phys. Rev. 115, 485 (1959).

54. Peskin, M. \& Tonomura, A. The Aharonov-Bohm effect Vol. 340 (Springer, Berlin, 1989).

55. Bezerra, V. B. Gravitational analogs of the Aharonov-Bohm effect. J. Math. Phys. 30, 2895 (1989).

56. Bohm, A., Mostafazadeh, A., Koizumi, H., Niu, Q. \& Zwanziger, J. The Geometric Phase in Quantum Systems: Foundations, Mathematical Concepts and Applications in Molecular and Condensed Matter Physics (Springer, New York, 2003).

57. Anacleto, M. A., Salako, I. G., Brito, F. A. \& Passos, E. Analogue Aharonov-Bohm effect in neo-Newtonian theory. Phys. Rev. D 92, 125010 (2015).

58. Khalilov, V. R. Bound states of massive fermions in Aharonov-Bohm-like fields. Eur. Phys. J. C 74, 2708 (2014).

59. Coste, C., Lund, F. \& Umeki, M. Scattering of dislocated wave fronts by vertical vorticity and the Aharonov-Bohm effect. I. Shallow water. Phys. Rev. E 60, 4908 (1999).

60. Vitória, R. L. L. \& Bakke, K. Aharonov-Bohm effect for bound states in relativistic scalar particle systems in a spacetime with a spacelike dislocation. Int. J. Mod. Phys. D 27, 1850005 (2018).

61. Vilenkin, A. Cosmic strings and domain walls. Phys. Rep. 121, 263 (1985).

62. de Marques, G. A., Furtado, C., Bezerra, V. B. \& Moraes, F. Landau levels in the presence of topological defects. J. Phys. A : Math Theor. 34, 5945 (2001).

63. Furtado, C. \& Moraes, F. Harmonic oscillator interacting with conical singularities. J. Phys. A : Math. Theor. 33, 5513 (2000).

64. Zare, S., Hassanabadi, H. \& de Montigny, M. Non-inertial effects on a generalized DKP oscillator in a cosmic string space-time. Gen. Relativ. Gravit. 52, 25 (2020).

65. Chrichfield, C. L. Scalar potentials in the Dirac equation. J. Math. Phys. 17, 261 (1976).

66. Hassanabadi, H., Zarrinkamar, S. \& Rahimov, H. Approximate Solution of D-Dimensional Klein-Gordon Equation with HulthénType Potential via SUSYQM. Commun. Theor. Phys. 53, 423 (2011).

67. Hassanabadi, H., Rahimov, H. \& Zarrinkamar, S. Cornell and Coulomb interactions for the D-dimensional Klein-Gordon equation. Ann. Phys. 523, 566 (2011).

68. Eshghi, M. \& Hamzavi, M. Yukawa-like confinement potential of a scalar particle in a Gödel-type spacetime with any l. Eur. Phys. J. C 78, 522 (2018).

69. de Montigny, M., Zare, S. \& Hassanabadi, H. Fermi field and Dirac oscillator in a Som-Raychaudhuri space-time. Gen. Relativ. Gravit. 50, 47 (2018).

70. Ikhdair, S. M. \& Sever, R. Relativistic and nonrelativistic bound states of the isotonic oscillator by Nikiforov-Uvarov method. J. Math. Phys. 52, 122108 (2011).

71. Ikhdair, S. M. \& Sever, R. Two approximation schemes to the bound states of the Dirac-Hulthén problem. J. Phys. A : Math. Theor. 44, 355301 (2011).

72. Fonseca, I. C. \& Bakke, K. On an atom with a magnetic quadrupole moment subject to harmonic and linear confining potentials. Proc. R. Soc. A 471, 20150362 (2015).

73. Ronveaux, A. Heun's Differential Equations (Oxford University Press, Oxford, 1995).

74. Slavyanov, S. Y. \& Lay, W. Special Functions: A Unified Theory Based in Singularities (Oxford University Press, New York, 2000). 
75. Arfken, G. B. \& Weber, H. J. Mathematical Methods For Physicists (Elsevier Academic Pres, London, 2005).

76. Witten, E. Search for a realistic Kaluz-Klein theory. Nuc. Phys. B 186, 412 (1981).

77. Trautman, A. Fibre bundles associated with space-time. Rep. Math. Phys. 1, 29 (1970).

78. Cho, Y. M. Higher-dimensional unifications of gravitation and gauge theories. J. Math. Phys. 16, 2029 (1975).

\section{Acknowledgements}

Author sincerely acknowledge the anonymous kind referee(s) for their value comments and suggestions which have greatly improved this present manuscript.

\section{Author contributions}

F.A. has done the whole work.

\section{Competing interests}

The author declares no competing interests.

\section{Additional information}

Correspondence and requests for materials should be addressed to F.A.

Reprints and permissions information is available at www.nature.com/reprints.

Publisher's note Springer Nature remains neutral with regard to jurisdictional claims in published maps and institutional affiliations.

Open Access This article is licensed under a Creative Commons Attribution 4.0 International

License, which permits use, sharing, adaptation, distribution and reproduction in any medium or format, as long as you give appropriate credit to the original author(s) and the source, provide a link to the Creative Commons licence, and indicate if changes were made. The images or other third party material in this article are included in the article's Creative Commons licence, unless indicated otherwise in a credit line to the material. If material is not included in the article's Creative Commons licence and your intended use is not permitted by statutory regulation or exceeds the permitted use, you will need to obtain permission directly from the copyright holder. To view a copy of this licence, visit http://creativecommons.org/licenses/by/4.0/.

(c) The Author(s) 2021 